\title{
Mixing of a wet granular medium: Effect of the particle size, the liquid and the granular compacity on the intensity consumption
}

\author{
Romain Collet $^{\mathrm{a}, \mathrm{b}, *}$, Driss Oulahna ${ }^{\mathrm{a}}$, Alain De Ryck ${ }^{\mathrm{a}}$, Pierre Henri Jezequel ${ }^{\mathrm{b}}$, Mylène Martin ${ }^{\mathrm{b}}$ \\ a RAPSODEE FRE-CNRS 3213, École des Mines d'Albi, Campus Jarlard, 81013 Albi, France \\ ${ }^{\mathrm{b}}$ LAFARGE Centre de Recherche, 95 rue de Montmurier - BP 15, F-38291 St. Quentin Fallavier, France \\ Corresponding author at Rapsodee (oulahna@mines-albi.fr)
}

A B S T R A C T

The mixing of a wetted insoluble granular medium was experimentally investigated focusing on the variation of the specific intensity consumption of mixing with addition of liquid in the granular bulk.

Preliminary results showed the influence of the particle average diameter of glass beads and calcites $\left(\mathrm{CaCO}_{3}\right)$, on the intensity consumption which decreased with the increase of the particles size (for the capillary and funicular states). However, an exception was observed at the level of the funicular state of the calcite, for which there was no particle size influence on the intensity consumption (for particles sizes inferior to $150 \mu \mathrm{m}$ ).

The effect of an inhomogeneous liquid distribution was not clearly demonstrated. However, the funicular intensity consumption was linked with the compacity of the medium.

By stopping the liquid flow at the end of the funicular state, and by continuing the mixing of a calcite, the funicular state intensity consumption and the measured granular compacities were increased. Moreover, this compacity measured during the funicular state, decreased with the particle size.

Wet granular mixing

Particle size

Liquid distribution

Granular compacity

Funicular state

\section{Introduction}

Mixing of wet granular media provides a homogeneous distribution of solids in the mixer and improves the wetting of the granular medium. However, it is a process that induces high energy consumption when the granular medium resists mixing (shearing).

In order to decrease the specific intensity consumption of mixing, a classification can be made of the factors (powders and liquid properties, process parameters) that have an effect on the inter-particular forces (capillary, Van-Der-Waals, gravitational, frictional, viscous forces, etc.) [1-3] and hence on the mixing efficiency.

The use of progressive addition, with a controlled flow, is worth studying because it leads to an increase in the time scale. The specific intensity consumption evolution with the added liquid amount (Fig. 1) characterise the mixing behaviour in different wetting states of the granular medium. The intensity profile may be divided in five sections [4-7]:

At the beginning of the mixing process (I), the first amount of liquid added is distributed at the surface of the particles without the formation of liquid bridges. Then, in the pendular state (II), the liquid amount at the particle surface is high enough to form capillary bridges between them, while the medium becomes more cohesive and there is a rapid increase in intensity consumption. During the funicular state (III), the porous volume of the previously formed agglomerates is filled by the additional liquid. In this state, the increase in the intensity is much lower because the majority of the capillary bridges have already been formed in the pendular state. When the porosity in the agglomerates is completely filled by the liquid (end of III), the additional liquid forms new liquid bridges between agglomerates until the formation of a single agglomerate. This is the capillary state (IV), characterised by the appearance of a rapid increase in intensity consumption (capillary peak). Finally, in the droplet state, the volume of liquid becomes higher than the inter-particle porosity of the granular medium and the intensity consumption decreases.

In a granular medium wetted by water, the dominant force is the cohesive capillary force " $F_{C}$ " [3]. This force depend on the particle diameter " $d$ ", on the liquid superficial tension " $\gamma_{L}$ ", and on a function that takes into account the contact angle " $\theta$ ", the filling angle of the liquid bridge " $\beta$ " (Fig. 2), and the ratio of the distance between particles with the particles average diameter " $a / d$ " $[2,8]$ :

$F_{C}=\gamma_{L} d f\left(\theta, \beta, \frac{a}{d}\right)$

A law can be used to take into account the cohesive forces " $F_{T}$ " in the shear resistance of a granular medium. This is the tensile strength Rumpf's law [9] which is defined for a regular packing of 


\begin{tabular}{|c|c|}
\hline \multicolumn{2}{|c|}{ Nomenclature } \\
\hline$a$ & distance between two particles (m) \\
\hline C & compacity of a granular medium (\%) \\
\hline$d$ & diameter of a particle $(\mu \mathrm{m})$ \\
\hline$d 50$ & mean diameter of a granular medium $(\mu \mathrm{m})$ \\
\hline$F_{C}$ & capillary force $(\mathrm{N})$ \\
\hline$F_{T}$ & cohesive forces $(\mathrm{N})$ \\
\hline$I_{M}$ & specific intensity $\left(\mathrm{A} \mathrm{kg}^{-1}\right)$ \\
\hline$m_{S}$ & mass of solids present in a granular medium (g) \\
\hline$V_{p}$ & porous volume of a granular medium $\left(\mathrm{cm}^{3}\right)$ \\
\hline$V_{R}$ & liquid/Solid volume ratio \\
\hline$V_{R, C}$ & liquid/Solid volume ratio of the capillary peak \\
\hline$V_{R, P}$ & $\begin{array}{l}\text { liquid/Solid volume ratio of the pendular state } \\
\text { beginning }\end{array}$ \\
\hline$V_{S}$ & volume of solid present in a granular medium $\left(\mathrm{cm}^{3}\right)$ \\
\hline$V_{T}$ & volume of a granular medium $\left(\mathrm{cm}^{3}\right)$ \\
\hline$\beta$ & filling angle of a liquid bridge $\left(^{\circ}\right)$ \\
\hline$\varepsilon$ & inter-particular porosity of a granular medium (\%) \\
\hline$\gamma_{L}$ & liquid superficial tension $\left(\mathrm{N} \mathrm{m}^{-1}\right)$ \\
\hline$\Pi$ & dimensionless liquid amount \\
\hline$\theta$ & liquid to solid contact angle $\left(^{\circ}\right)$ \\
\hline$\rho$ & bulk density $\left(\mathrm{g} \mathrm{cm}^{-3}\right)$ \\
\hline$\rho_{A}$ & aerated bulk density $\left(\mathrm{g} \mathrm{cm}^{-3}\right)$ \\
\hline$\rho_{S}$ & true density $\left(\mathrm{g} \mathrm{cm}^{-3}\right)$ \\
\hline$\rho_{T}$ & tapped bulk density $\left(\mathrm{g} \mathrm{cm}^{-3}\right)$ \\
\hline$\sigma_{T}$ & tensile strength $(\mathrm{Pa})$ \\
\hline$\sigma_{T, C}$ & tensile strength of a liquid bridge (Pa) \\
\hline
\end{tabular}

monomodal spheres:

$\sigma_{T}=\frac{(1-\varepsilon)}{\varepsilon} \frac{F_{T}}{d^{2}}$

The Eqs. (1) and (2) give the Rumpf's law of a granular medium which is proportional to the inverse of the average particle size [8]:

$\sigma_{T, C}=\frac{(1-\varepsilon)}{\varepsilon} \frac{\gamma_{L}}{d} f\left(\theta, \beta, \frac{a}{d}\right)$

This law also takes into account the inter-particular porosity " $\varepsilon$ " of the granular packing. Several authors have shown that addition of liquid in a dry granular medium modifies the compacity [10-14]. The porosity increases in the pendular state before remaining constant in the funicular state. Moreover, the porosity measured in the funicular state increases with the decrease of the particles size. Adding liquid will lead to the formation of liquid bridges between

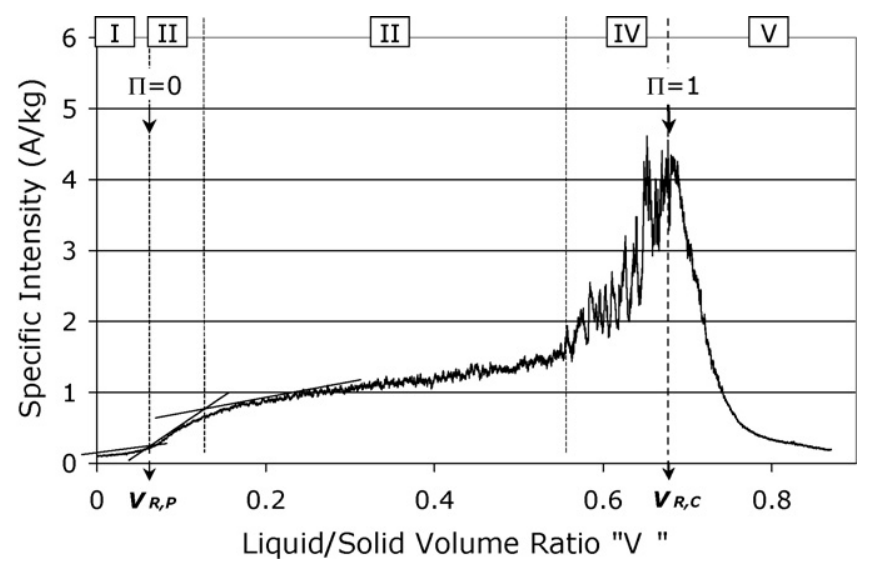

Fig. 1. Intensity consumption profile: mixing of the calcite 2 with a graduated liquid addition $(40 \mathrm{ml} / \mathrm{min})$.

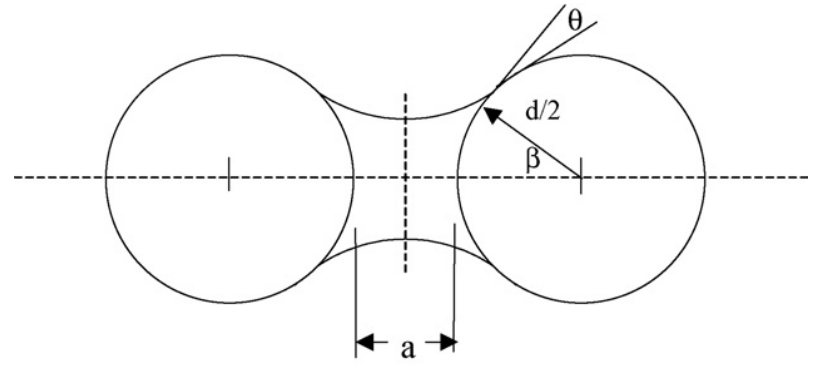

Fig. 2. Liquid bridge between two spheres.

particles and generate a capillary force limiting the relative motion of particles and giving high porosity. The increase of porosity is more pronounced for small than for large particles as a result of an increased capillary force relative to gravity. So, the inter-granular porosity is probably modified and must be taken into account during the mixing.

This paper presents firstly the influence of the particle average diameter on the intensity consumed during the mixing at the levels of the funicular and capillary state. "Two model granular materials were used in this study: glass beads (spherical, monomodal granular distribution, no reactivity with water) and calcites (non-spherical, monomodal granular distribution, no reactivity with water) allowing the observation of different behaviours during mixing."

The second part focuses on the different behaviours present in the funicular state.

\section{Experimental set up and materials}

The mixer used was a planetary mixer from the $\mathrm{CAD}^{\circledR}$ company (Mlx60). It can be used to make a hard paste, and it allows the intensity consumption of the engine to be recorded, at a frequency of 10 points per second. The available rotation velocity is between 0 and $300 \mathrm{rpm}$ and the volume of the bowl is $4 \mathrm{~L}$.

A preliminary study allowed the optimal process parameters to be set: an impeller speed of $120 \mathrm{rpm}$, a droplet-by-droplet liquid flow rate of $40 \mathrm{ml} / \mathrm{min}$, and a powder volume " $V_{T}$ " of $1250 \mathrm{~cm}^{3}$ (This volume take into account the volume of solid " $V_{S}$ " and the inter-particle porous volume " $V_{P}$ "). The liquid was added using a peristaltic pump and a feeder pipe, $3 \mathrm{~mm}$ in diameter. Moreover, the necessary mass of powder " $m_{S}$ " filling $1250 \mathrm{~cm}^{3}$ was determined using the bulk density of the granular medium " $\rho$ ", equal to the average of the aerated " $\rho_{A}$ " and tapped " $\rho_{T}$ " densities:

$\rho=\frac{m_{S}}{V_{T}}=\frac{m_{S}}{V_{S}+V_{P}}$

$m_{S}=1250 \rho=1250 \frac{\rho_{A}+\rho_{T}}{2}$

Materials used (Table 1) were spherical glass beads supplied by SOVITEC ${ }^{\circledR}$ and calcites supplied by OMYA ${ }^{\circledR}$. The volume granulometric distributions were obtained with a MALVERN ${ }^{\circledR}$ Mastersizer 2000 , the true density " $\rho_{S}$ " of the solids with a helium picnometer (Accupyc 1330-MICROMERITIC ${ }^{\circledR}$ ), and the dry porosities " $\varepsilon$ " (or the compacity " $C$ ") using the values of the true " $\rho_{S}$ " and bulk " $\rho$ " densities:

$\rho_{S}=\frac{m_{S}}{V_{S}}$

$\varepsilon=1-\frac{\rho}{\rho_{S}}=1-C$

The liquid used for all the tests was water. 
Table 1

Material properties.

\begin{tabular}{|c|c|c|c|c|c|c|}
\hline & $d 10(\mu \mathrm{m})$ & $d 50(\mu \mathrm{m})$ & $d 90(\mu \mathrm{m})$ & True density $\rho_{S}\left(\mathrm{~g} / \mathrm{cm}^{3}\right)$ & Bulk density $\rho\left(\mathrm{g} / \mathrm{cm}^{3}\right)$ & Porosity $\varepsilon(\%)$ (dry state) \\
\hline Calcite 1 & 1 & 6 & 17 & 2.73 & 0.87 & 68 \\
\hline Calcite 2 & 2 & 18 & 47 & 2.73 & 1.13 & 58 \\
\hline Calcite 3 & 3 & 29 & 68 & 2.73 & 1.32 & 51 \\
\hline Calcite 4 & 58 & 90 & 140 & 2.73 & 1.36 & 50 \\
\hline Calcite 5 & 102 & 156 & 225 & 2.73 & 1.36 & 50 \\
\hline Calcite 6 & 140 & 220 & 317 & 2.73 & 1.39 & 49 \\
\hline Calcite 7 & 181 & 258 & 354 & 2.73 & 1.38 & 49 \\
\hline Calcite 8 & 316 & 524 & 803 & 2.72 & 1.45 & 47 \\
\hline Glass beads 1 & 18 & 30 & 48 & 2.48 & 1.44 & 42 \\
\hline Glass beads 2 & 64 & 88 & 122 & 2.51 & 1.49 & 41 \\
\hline Glass beads 3 & 117 & 160 & 219 & 2.52 & 1.49 & 41 \\
\hline Glass beads 4 & 194 & 261 & 353 & 2.52 & 1.46 & 42 \\
\hline Glass beads 5 & 391 & 538 & 738 & 2.52 & 1.49 & 41 \\
\hline
\end{tabular}

\section{Results and discussion}

\subsection{Particle size effect on the specific intensity consumption of} mixing in funicular and capillary states

Several glass beads and calcites were mixed in order to study the solids properties effect on the granular medium behaviour during the mixing (shear resistance, wet inter-particular porosity). The measurements of the intensity consumed at the levels of funicular and capillary states are presented as a function of the inverse of the mean particles size (Fig. 3). This representation takes into account the Rumpf's law for which the tensile strength is proportional to the inverse of the particle size.

Firstly, the calcites generate higher intensity consumption than the glass beads. The different particle shapes of the two materials are responsible for this difference in mixing resistance. The calcites are less spherical than the glass beads and generate additional frictional forces that increase the shear resistance $[15,16]$.

The intensity increases linearly with $1 / d 50$ for $d 50<150 \mu \mathrm{m}$. This result shows that the shear resistance (or the mixing resistance) is linked to the specific surface of particles. However, the $y$-intercept is not equal to zero. This difference with the Rumpf's law shows that the conditions for which this model is defined are not fulfilled. The mixing is achieved in a dynamic regime and the calcites are composed of irregular shapes non-monomodal particles whereas the Rumpf's law is defined in a static regime for spherical monomodal particles. On the other hand, this phenomenon is also caused by the consideration of the specific intensity consumption measured in the dry state, before liquid addition.

There is an exception for the funicular state of the calcites for which there is not a particle size effect (for $d 50<150 \mu \mathrm{m}$ ). To explain this stability of the intensity with particle size, our hypotheses are an inhomogeneously liquid distribution through the granular medium and/or an increase of the granular porosity when particle size decreases.

\subsection{Independence of the funicular intensity consumption with the calcites particle size}

To study the independence of the funicular intensity consumption with the calcites particle size, different approaches were used to characterise the liquid distribution and the granular compacity:

- Measurement of the liquid content present in several wet granular samples

- Tests carried out in order to increase the intensity consumption of the funicular state

- Modification of the liquid flow and the number of liquid feeds

- Mixing after stopping the liquid flow: observation of the intensity evolution and porosity measurements of granular samples removed, just after stopping the liquid flow and $20 \mathrm{~min}$ later

- Inter-particular porosity measurements of granular samples removed during the mixing, from the dry to the capillary state

\subsubsection{Measurement of liquid content present in several wet} granular samples

In order to measure the liquid content present in several wet samples, three calcites with a mean diameter higher or lower than $150 \mu \mathrm{m}$ were used (calcite 3, 4 and 7; Table 1 ). The mixing (liquid flow of $40 \mathrm{ml} / \mathrm{min}$, rotation velocity of $120 \mathrm{rpm}$ ) was stopped in the funicular state in order to take five wet granular samples $\left(\approx 10 \mathrm{~cm}^{3}\right)$ in different places of the bowl (in the middle or at the wall). With the masses of the wet and dried $\left(24 \mathrm{~h}\right.$ at $\left.100^{\circ} \mathrm{C}\right)$ samples, the liquid content present in every sample could be determined and compared with the liquid amount really added in the mixer. The results show that the liquid content added in the mixer and present in every sample are similar. So, at the scale of our samples, the liquid amount is localized homogeneously. However, even if an inhomogeneous liquid distribution is not observed at the scale of the samples, lack of homogeneity could be present at the scale of the agglomerates.

\subsubsection{Tests carried out to increase the intensity consumption of the funicular state}

The following tests were achieved in order to improve the liquid distribution and/or the granular compacity.

3.2.2.1. Modification of the liquid flow and the number of liquid feeds. The number of liquid feeds was increased with keeping a constant liquid flow of $40 \mathrm{ml} / \mathrm{min}$. The calcite 2 , composed of particles lower than $150 \mu \mathrm{m}$, was mixed firstly with one liquid feed delivering $40 \mathrm{ml} / \mathrm{min}$, and then with three liquid feeds spaced out $120^{\circ}$, each delivering $13.3 \mathrm{ml} / \mathrm{min}$.

The results show that there is no effect of the number of liquid feeds. Indeed, the signals of the intensity consumption, obtained with one and three liquid feeds, are perfectly superposed. This test shows that the mixer seems to realize the same liquid distribution whatever the number of the liquid feeds used, and particularly for the smaller calcites like the calcite 2 .

Liquid flows between 10 and $80 \mathrm{ml} / \mathrm{min}$ were achieved for the mixing of the calcite 1 . The intensity consumptions obtained in the funicular state increase lightly with the liquid flow but this effect is too small to conclude that the liquid flow has an effect on the liquid distribution.

3.2.2.2. Mixing after stopping the liquid flow. In order to study the liquid distribution and the granular compacity during the mixing, the liquid flow $(40 \mathrm{ml} / \mathrm{min})$ was stopped in the funicular state of the calcite 2 and the glass beads 1 , and the mixing was maintained during $20 \mathrm{~min}$. The intensity consumption evolution was observed, 

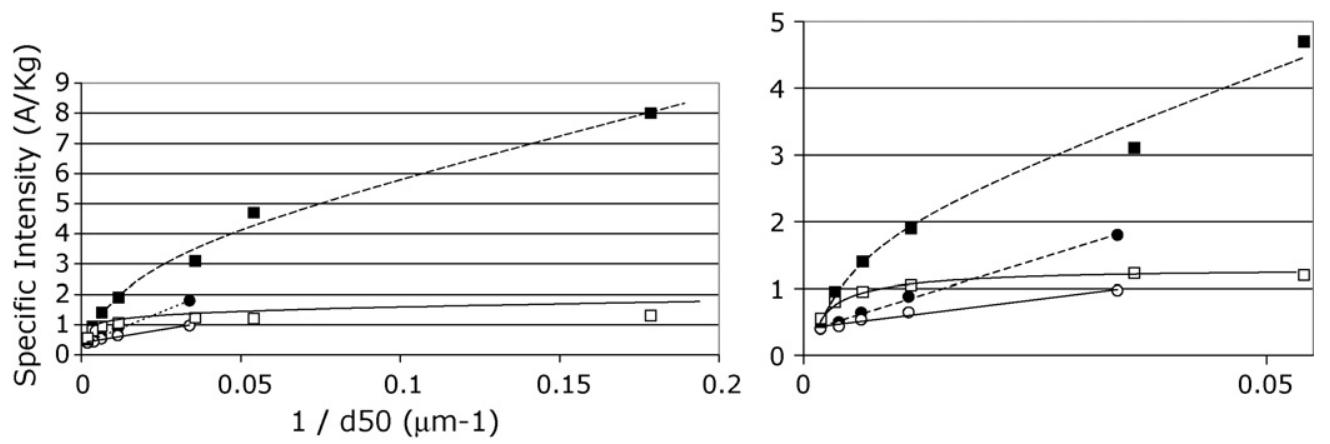

- Capillairy State - Glass Beads - o Funicular State
- Capillary State - Calcites - $\quad$ 口Funicular State

Fig. 3. Specific intensity consumption of mixing for the glass beads and the calcites as a function of the inverse of the mean particle size (1/d50).

after the stop of the liquid flow (Fig. 4). Tests were carried out with a stop of the liquid flow at the middle and at the end of the funicular state.

In addition, the porosity of several granular samples was measured just after stopping the liquid flow and after 20 min of mixing without flow (Table 2). Three different tubular punches (1570, 2260 and $9120 \mathrm{~mm}^{3}$ ) allowed making the samples, and for every punch volume $\left(V_{T, E}\right)$, three samples were taken. Then, all the sample were dried in a drying oven $\left(100^{\circ} \mathrm{C}\right.$ during $\left.24 \mathrm{~h}\right)$ and the porosity " $\varepsilon$ " was determined with the following equation:

$\varepsilon=1-\frac{m_{S}}{V_{T, E} \rho_{S}}$

For the flow stopped at the middle of the calcite funicular state, the intensity consumptions and the porosities determined after stopping the liquid flow and $20 \mathrm{~min}$ later are the same. In this case, stopping the liquid flow has no effect as there is not enough liquid in the system to allow a reorganization of the wet granular medium.

After stopping the liquid flow at the end of the calcite funicular state, the intensity increases (from 1.4 to $2.1 \mathrm{~A} \mathrm{~kg}^{-1}$ ) and the porosity decreases (from $68 \%$ to $42 \%$ ) with the mixing time. These results show that the liquid distribution in the calcite granular medium is not complete at the end of the funicular state, when the liquid is added progressively. So, an additional mixing time improves the liquid distribution, and consequently increases the intensity consumption and the compacity.

The intensity consumption and the compacity of the glass beads remain constant after stopping the liquid flow at the end or in the middle of funicular state. This result shows that glass beads do not need more mixing time to distribute the liquid and to reach the maximal compacity, contrary to the calcite.

These first results confirm our hypotheses explaining the independence of the funicular intensity consumption with the calcites particle size. In the funicular regime, the liquid distribution seems to be incomplete. Furthermore, the inter-particular porosity is very important in this state and decrease with the mixing time after stopping the liquid flow.

In the next part, it will be shown that the inter-particular porosity in the funicular state increases when the particle diameter decreases.

\subsubsection{Inter-particular porosity of granular samples removed} during the mixing

Using the method described in Section 3.2.2.2, measurements of porosity were achieved for several glass beads and calcites granular samples. These samples were taken during the mixing for different liquid saturation rates, from the dry state to the capillary state. In order to calculate corresponding amounts of liquid for the different solids, we used the dimensionless amount of liquid $\Pi$ proposed by Leuenberger et al. [17]:

$\Pi=\frac{V_{R}-V_{R, P}}{V_{R, C}-V_{R, P}}$

" $V_{R}$ " is the liquid/solid volume ratio, " $V_{R, P}$ " is situated at the beginning of the pendular state, and " $V_{R, C}$ " correspond to the capillary state (Fig. 1).

Figs. 5 and 6 show that the two materials present similar porosity evolutions. From the dry $(\Pi=0)$ to the wet state $(\Pi=0.1)$, the
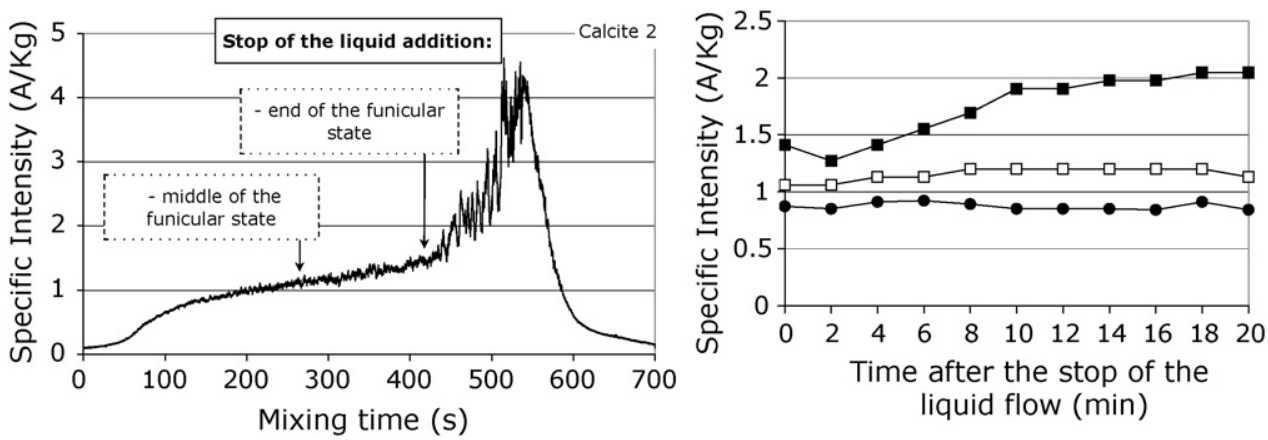

$$
\begin{aligned}
& \text { - Middle of the calcite } 2 \text { funicular state } \\
& - \text { End of the calcite } 2 \text { funicular state } \\
& - \text { End of the glass beads } 1 \text { funicular state }
\end{aligned}
$$

Fig. 4. Evolution of the specific intensity consumption of mixing after stopping the liquid flow in the funicular state. 
Table 2

Inter-granular porosities of granular samples taken after having stopped the liquid flow and after 20 min of mixing without flow.

\begin{tabular}{|c|c|c|c|c|c|}
\hline & \multirow[t]{3}{*}{ Dry initial state } & \multirow{2}{*}{\multicolumn{2}{|c|}{$\frac{\text { Middle of the funicular state }}{\text { Time after stopping the liquid flow (min) }}$}} & \multicolumn{2}{|c|}{ End of the funicular state } \\
\hline & & & & & \\
\hline & & 0 & 20 & 0 & 20 \\
\hline & 1 & 2 & 3 & 4 & 5 \\
\hline Porosity of calcite 2 (\%) & 59 & $75 \pm 3$ & $74 \pm 2$ & $68 \pm 4$ & $42 \pm 3$ \\
\hline Porosity of glass beads 1 (\%) & 42 & $59 \pm 6$ & - & $54 \pm 3$ & $54 \pm 4$ \\
\hline
\end{tabular}

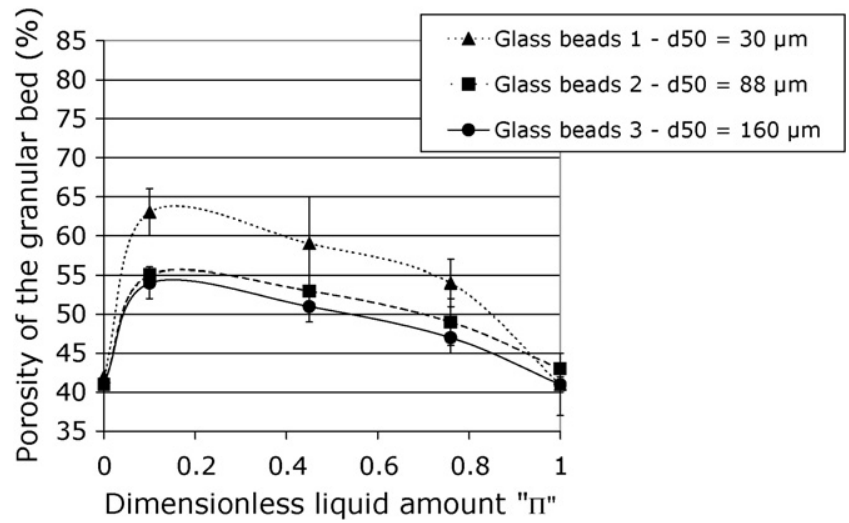

Fig. 5. Evolution of the porosity of glass beads during mixing.

porosity increases (in the pendular state) before remaining constant in the funicular state $(0.1<\Pi<0.75)$. Then, in the capillary state $(\Pi>0.75)$, it decreases. Moreover, the porosity determined in the funicular state increases with the decrease of the particle size. These observations are in agreement with previous works [10-14] described in Section 1.

However, some differences are observed between the solids. For the bigger particles (glass beads, calcites 4 and 5), the initial porosity (dry state) is the same as the final porosity (capillary state). In this case, the porosities of the calcites are higher than the porosities of the glass beads because of the calcites are less spherical [18]. For the smaller particles (calcite 1-3), the final porosity is lower than the initial porosity. Under a critical particle size, the dry porosity increases with a decrease of the particle size because of the effect of the Van-Der-Waals cohesive forces: a restriction of the relative motion of particles [10-13].

In the funicular state, the porosity of the calcites increases with the decrease of the particle size, to attain values between $70 \%$ and $80 \%$ for the smaller sizes (under $30 \mu \mathrm{m}$ ). This induces a decrease of the density of contact points and of the frictional and capillary cohesive forces (in a wet state, i.e. pendulary, funicular or capillary

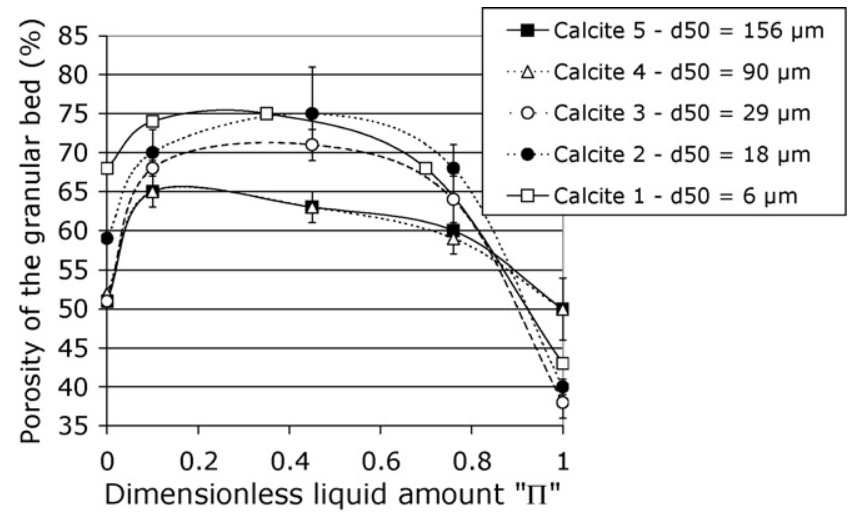

Fig. 6. Evolution of the porosity of calcites during mixing. state, the Van-Der-Waals cohesive forces are negligible compared to capillary forces).

\section{Conclusion}

The mixing of two different wetted insoluble granular media (non-spherical calcite and glass beads) was experimentally studied focusing on the variation of the specific intensity consumption of mixing with addition of liquid in the granular bulk.

According to the Rumpf's law, we firstly showed that the specific intensity consumption is function of the inverse of the particles size (for the capillary and funicular states). However, an exception was observed at the level of the funicular state of the calcite, for which there is no particle size influence on the specific intensity consumption.

By stopping the liquid flow at the end of the funicular state, and by continuing the mixing of a calcite, the specific intensity consumption and the granular compacities were increased. On the contrary, the intensity and the compacity of glass beads remained constant after the stop of the liquid flow. These phenomena explain partly the stability of the calcite funicular intensity when particle size decreases. The liquid distribution and the compacity are incomplete in the funicular state and need more mixing time to be improved.

Moreover, measurements of calcites granular porosities during the mixing (from the dry to the capillary state) showed that the porosity of the funicular state increases until $70-80 \%$ with the decrease of the particles size. So, the space between particles is increased and the inter-particle forces (capillary and frictional) are decreased consequently. This phenomenon allows keeping the specific intensity consumption in the funicular state constant.

Otherwise, these measurements during the mixing show that the porosity is not constant from the dry to the capillary state. A dilation of the granular bed takes place in the pendular state, and a densification occurs in the capillary state. So, the compacity depends on the added liquid amount, influences the shear resistance (and consequently the specific intensity consumed by the mixer), and is an important parameter to take into account in the study of the granular medium behaviour during mixing.

\section{References}

[1] J.Q. Feng, D.A. Hays, Relative importance of electrostatic forces on powder particles, Powder Technology 135-136 (2003) 65-75.

[2] J.P.K. Seville, C.D. Willett, P.C. Knight, Interparticle forces in fluidisation: a review, Powder Technology 113 (2000) 261-268.

[3] S.M. Iveson, J.A. Beathe, N.W. Page, The dynamic strength of partially saturated powder compacts: the effect of liquid properties, Powder Technology 127 (2002) 149-161.

[4] G. Betz, P. Junker Bürgin, H. Leuenberger, Power consumption profile analysis and tensile strength measurements during moist agglomeration, International Journal of Pharmaceutics 252 (2003) 11-25.

[5] G. Betz, P.J. Bürgin, H. Leuenberger, Power consumption measurement and temperature recording during granulation, International Journal of Pharmaceutics 272 (2004) 137-149.

[6] A. Goldszal, J. Bousquet, Wet agglomeration of powders: from physics toward process optimization, Powder Technology 117 (2001) 221-231.

[7] B. Cazacliu, J. Legrand, Characterization of the granular-to-fluid state process during mixing by power evolution in a planetary concrete mixer, Chemical Engineering Science 63 (2008) 4617-4630. 
[8] P. Pierrat, H.S. Caram, Tensile strength of wet granular materials, Powder Technology 91 (1997) 83-93.

[9] H. Rumpf, Haftung und festigkeit von agglomeraten, Die Pharmaceutische Industrie 34 (1972) 270-281.

[10] C.L. Feng, A.B. Yu, Effect of liquid addition on the packing of mono-sized coarse spheres, Powder Technology 99 (1998) 22-28.

[11] C.L. Feng, A.B. Yu, Quantification of the relationship between porosity and interparticle forces for the packing of wet uniform spheres, Journal of Colloid and Interface Science 231 (2000) 136-142.

[12] A.B. Yu, N. Standish, L. Lu, Coal agglomeration and its effect on bulk density, Powder Technology 82 (1995) 177-189.

[13] A.B. Yu, C.L. Feng, R.P. Zou, R.Y. Yang, On the relationship between porosity and inter-particle forces, Powder Technology 130 (2003) 70-76.
[14] E. Rondet, M. Delalonde, T. Ruiz, J.P. Desfours, Identification of granular compactness during the kneading of a humidified cohesive powder, Powder Technology 191 (2009) 7-12.

15] R. Collet, D. Oulahna, P.-H. Jezequel, A. de Ryck, M. Martin, Mixing of a wet granular medium: solid properties effects, in: 12th SFGP Congress, 14-16 October Marseille, France, 2009.

[16] R. Collet, Critères de malaxabilité des mélanges granulaires humides, PhD Thesis, Université de Toulouse, France, 2010.

[17] H. Leuenberger M. Puchkov, E. Krausbauer, G. Betz, Manufacturing pharmaceutical granules: is the granulation end-point a myth? Powder Technology 189 (2009) 141-148.

[18] D.J. Cumberland, R.J. Crawford, The Packing of Particles. Handbook of Powder Technology, vol. 6, Elsevier, 1987, pp. 99-117. 